\section{Cylindrical EBG antenna for short range gigabit wireless communications at millimetre-wave bands}

\author{
Y. Lee, X. Lu, Y. Hao, S. Yang, C.G. Parini and J.R.G. Evans
}

An omnidirectional millimetre-wave antenna based on a cylindrical electromagnetic bandgap (EBG) resonator is presented. The cylindrical woodpile is fabricated using the ceramic extrusion freeforming technique with high purity alumina powder. The designed antenna can achieve a narrow beam with $6.5^{\circ}$ measured half power beam-width in the elevation plane without using an array configuration. The overall antenna gain is approximately $5 \mathrm{dBi}$ at $94.2 \mathrm{GHz}$, suitable for future short range gigabit wireless communications.

Introduction: Owing to increasing demand for broadband, high data rate wireless systems, millimetre-wave bands are gaining more attention The frequency spectrum around $60 \mathrm{GHz}$ is of special interest for the high data rate wireless local area network (WLAN) because of the high absorption and attenuation in the atmosphere making this frequency band suitable for short range communications. A point-to-point, wireless communication link at the $92-95 \mathrm{GHz}$ band can provide data transmission rates of more than $1 \mathrm{Gbit} / \mathrm{s}$ at ranges of several miles during normal weather conditions. Recently, we have demonstrated a low profile high directivity antenna for millimetre-wave bands using a planar woodpile EBG structure [1]. In this Letter, we extend the idea to realise an EBG antenna based on cylindrical woodpiles. The proposed antenna is anticipated to exhibit an ominidirectional radiation pattern in the azimuth plane but highly directive in the elevation plane. Such an antenna has superior properties over conventional conformal antenna arrays because of its low profile and cost-effectiveness. Similar configurations using metallic elements for EBG structures have been studied and tested at microwave frequencies $[2,3]$; however, metallic elements are not preferred at millimetre-wave frequencies owing to their conduction losses by a small skin depth. In this work, we designed and fabricated a cylindrical woodpile resonator based on low-loss ceramic materials at $95 \mathrm{GHz}$, and demonstrated its use in antennas for short range gigabit wireless communications.

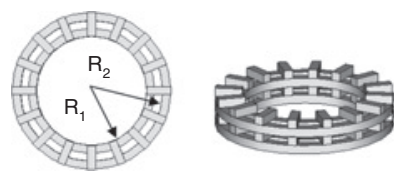

a

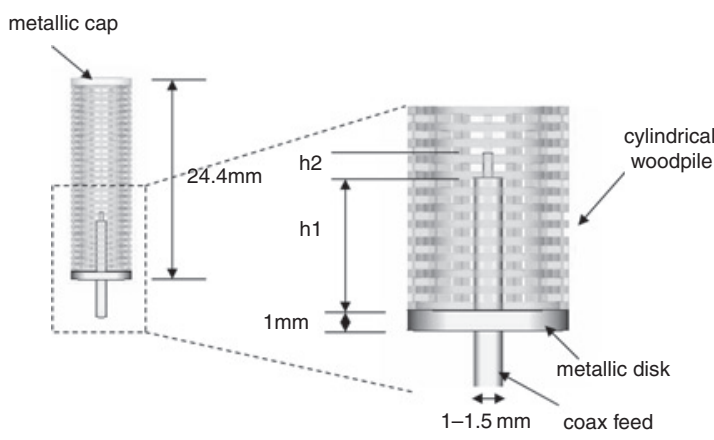

Fig. 1 Geometry of cylindrical woodpile EBG structure and cylindrical EBG resonator antenn

$a$ Cylindrical woodpile EBG structure

$b$ Cylindrical EBG resonator antenna

Cylindrical woodpile EBG design: We apply a similar geometry as in the planar woodpile structure [4] for the design of cylindrical woodpile EBGs made of alumina materials $\left(\varepsilon_{r}=9.6\right)$ with the filament diameter, $w=0.41 \mathrm{~mm}$. For the cylindrical woodpile cavity, the horizontal filaments are not interleaved, but aligned at the same horizontal location. As the constructed cylindrical cavity will be excited by vertical (axis of the cylinder) electric fields, it is essential to have vertically aligned gratings which are implemented by stacked horizontal filaments crossing at the same location. Fig. $1 a$ shows a cross-section of the cylindrical woodpile cylinder with two layers of rings having different diameters. The distance between the two rings determines the bandgap frequency of the cylindrical EBG. It has been found that the resonant frequencies of the cylindrical woodpile cavity are similar to those of conventional metallic cylindrical cavities. To create a narrow beam along the elevation plane, we need to use $T M_{0 \mathrm{n}}^{z}$ type modes propagating along the axis of the cylinder. To excite the fundamental mode at $95 \mathrm{GHz}$, a very small inner diameter $\left(\mathrm{R}_{1} \simeq 1 \mathrm{~mm}\right)$ is required for the cylindrical cavity, thus we designed the cylindrical woodpile to operate for higher modes in the radial direction. Fig. $1 b$ shows the initial design of the cylindrical woodpile resonator with coax feed operating around $95 \mathrm{GHz}$. The electric field inside the cavity does not show a phase variation along the $z$-direction (axis of the cylinder) but gradually tapers out towards the end of the cylinder, creating a uniform illumination of the field on the surface of the cylindrical woodpile. Cylinders with even larger diameters may be used, but owing to the diffraction and leakage from the truncated end of the cylinder, the sidelobe levels will be increased. However, this can be improved by using longer EBG cylinders. The resonant frequency of the EBG cylinder can be modified by changing the radius. Fig. 2 shows the simulated resonant frequency and directivity of the antenna against the radius of the cylinder.

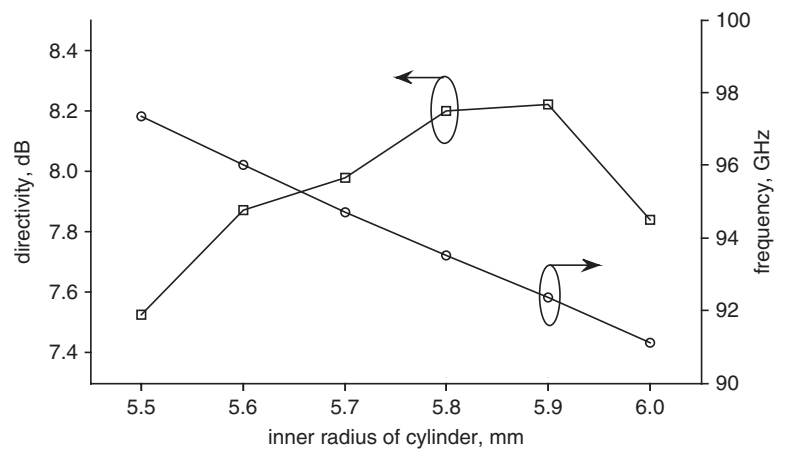

Fig. 2 Simulated directivity and resonant frequency of antenna against inner radius of cylindrical resonator (simulation of woodpile cylinder with ideal dipole source inside)

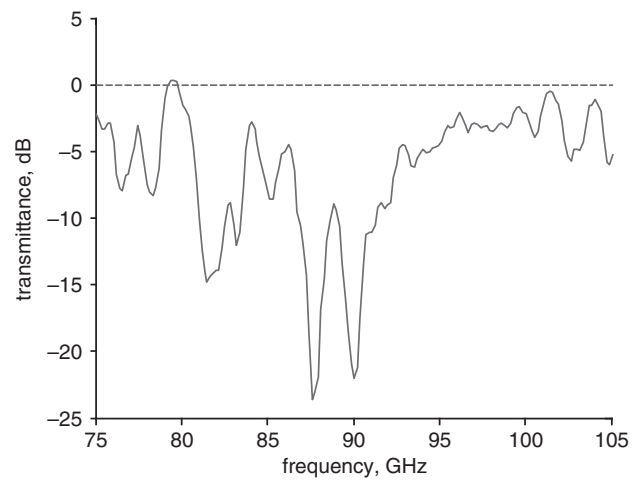

Fig. 3 Transmittance of cylindrical woodpile

Experimental results: The cylindrical woodpile structure has been fabricated using the extrusion freeforming facilities at Queen Mary University of London (QMUL). High purity alumina powder was used as a dielectric material and prepared as described in $[4,5]$. The inner and outer radii of the designed woodpile are $\mathrm{R}_{1}=2.75 \mathrm{~mm}$ and $\mathrm{R}_{2}=3.55 \mathrm{~mm}$, respectively. The bandgap of the fabricated woodpile EBG has been identified using a coax probe inside the cylinder and a waveguide horn outside the cylinder. The measured transmittance through the cylindrical woodpile is shown in Fig. 3. A stop band is observed between 85 and $95 \mathrm{GHz}$. To excite the cylindrical woodpile cavity, a monopole type feed was designed. Details of its design are shown in Fig. $1 b$. The location of the monopole $\left(\mathrm{h}_{1}\right)$ inside the cavity must be properly chosen because the overall geometry is not symmetrical owing to the presence of the monopole coax. It was noticed that the location of the feed should be slightly lower than the centre of the cylinder. The length of the monopole $\left(\mathrm{h}_{2}\right)$ can be tuned to achieve the impedance matching for the EBG antenna. The values of $h_{1}$ and $h_{2}$ for the prototype antenna are chosen as 4 and $1.4 \mathrm{~mm}$, respectively, while the 
height of the fabricated cylinder is $24 \mathrm{~mm}$. The effect of the metallic cap is minor for the designed woodpile cylinder, but it will be necessary for the cylinders with a larger diameter, if one wishes to reduce the radiation along the axis of the cylinder.

The designed antenna has been fabricated using a $1 \mathrm{~mm}$ coax feed and a waveguide (WR-10) to coax transition made in-house. The cylindrical woodpile was integrated with the coax feed using a brass circular disk with thickness of $1 \mathrm{~mm}$ and attached to the coax. The top of the cylindrical woodpile was terminated with thin aluminium foil. Details of the design parameters are shown in Fig. $1 b$. The fabricated antenna was measured in an anechoic chamber and the measured antenna patterns are shown in Fig. 4. A narrow beam is observed in the horizontal plane (i.e. elevation $=0^{\circ}$ ) with some sidelobes; however, large fluctuation was observed in the azimuth pattern because of a non-symmetry of the fabricated woodpile cylinder. The structural deformation is attributed to the nature of the fabrication method. For the designed woodpile cylinder, the length (height) of the cylinder is much greater than the diameter, thus sagging of soft filaments is introduced during the fabrication, which accumulates and results in variation of location of the cylinder axis at different heights of the cylinder. The HPBW of the prototype antenna in the elevation plane is $6.8^{\circ}$ and the measured gain is approximately $5 \mathrm{dBi}$. The gain is slightly reduced compared to the expected directivity owing to the mismatch between the cylindrical woodpile cavity and the feed.
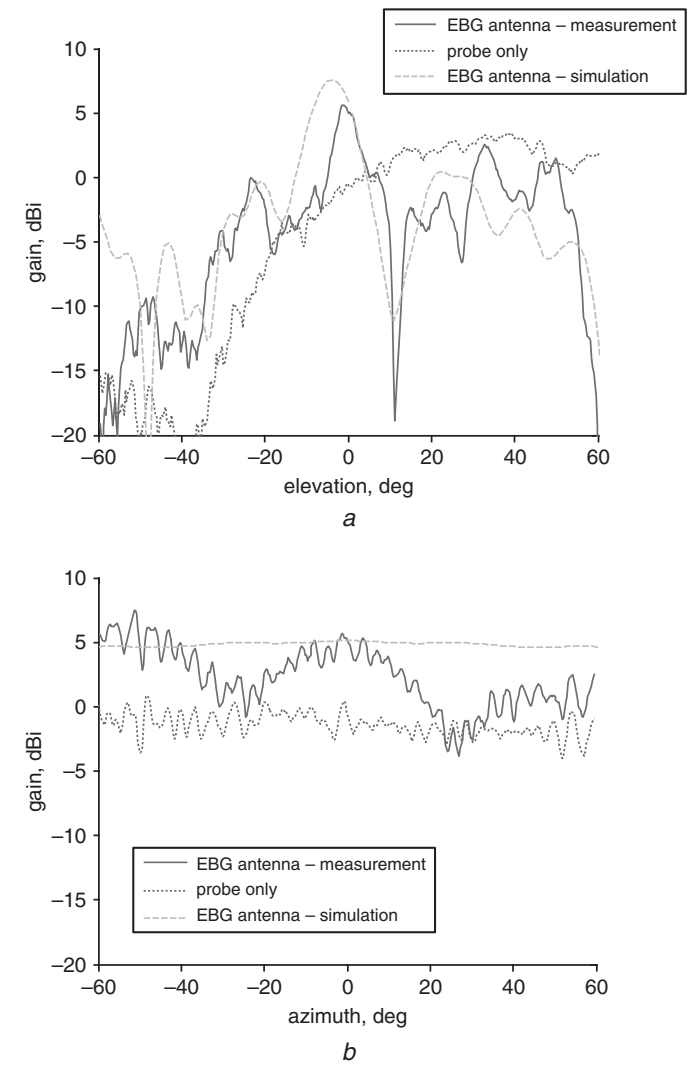

Fig. 4 Measured antenna pattern of millimetre-wave cylindrical woodpile EBG resonator antenna at $94.2 \mathrm{GHz}$ : elevation and azimuth

$a$ Elevation

$b$ Azimuth
Conclusion: We present an azimuthally omnidirectional antenna design based on the cylindrical woodpile EBG structure for applications at millimetre-wave frequencies. The cylindrical woodpile resonator achieves a narrow beam with improved gain. The designed cylindrical EBG structure has been fabricated using the ceramic extrusion freeforming technique. Experimental results confirmed that the designed antenna showed narrow beam in the horizontal plane, making the antenna useful for short range gigabit wireless communications at millimetre-wave frequencies.

Acknowledgment: The authors thank the Leverhulme Trust (grant no. $\mathrm{F} / 07476 / \mathrm{V}$ ) for supporting this research.

(C) The Institution of Engineering and Technology 2009 6 November 2008

Electronics Letters online no: 20093192

doi: 10.1049/el:20093192

Y. Lee, Y. Hao and C.G. Parini (School of Electronic Engineering and Computer Science, Queen Mary University of London, London, United Kingdom)

E-mail: yoonjae.lee@elec.qmul.ac.uk

X. Lu and J.R.G. Evans (Department of Chemistry, University College London, 20 Gordon Street, London WC1H0AJ, United Kingdom)

S. Yang (School of Engineering and Materials Science, Queen Mary University of London, London, United Kingdom)

\section{References}

1 Lee, Y., Lu, X., Hao, Y., Ubic, R., Evans, J.R.G., and Parini, C.G.: 'A directive millimetre-wave antenna based on freeformed woodpile EBG structure', Electron. Lett., 2007, 43, (4), pp. 195-196

2 Palikaras, G.K., Feresidis, A.P., and Vardaxoglou, J.C.: 'Cylindrical EBG surfaces for omni-directional wireless LAN antennas'. IEEE Int. Symp. Antennas and Propagation, Washington, DC, USA, July 2005, Vol. 4B, pp. 339-342

3 Chreim, H., Pointereau, E., Jecko, B., and Dufrane, P.: 'Omnidirectional electromagnetic band gap antenna for base station application', IEEE Antennas Wirel. Propag. Lett., 2007, 6, pp. 499-502

4 Lu, X., Lee, Y., Yang, S., Hao, Y., Ubic, R., Evans, J.R.G., and Parini, C.G.: 'Fabrication of electromagnetic crystals by extrusion freeforming', Metamaterials, 2007, 2, (1), pp. 36-44

5 Lee, Y., Lu, X., Hao, Y., Ubic, R., Evans, J.R.G., and Parini, C.G.: 'Rapid prototyping of ceramic millimetre-wave metamaterials: simulations and experiments', Microw. Opt. Technol. Lett., 2007, 29, (9), pp. 2090-2093 